\title{
ON THE CONJECTURE OF JEŚMANOWICZ CONCERNING PYTHAGOREAN TRIPLES
}

\author{
TAKAFUMI MIYAZAKI
}

\author{
(Received 28 November 2008)
}

\begin{abstract}
Let $a, b, c$ be relatively prime positive integers such that $a^{2}+b^{2}=c^{2}$ with $b$ even. In 1956 Jeśmanowicz conjectured that the equation $a^{x}+b^{y}=c^{z}$ has no solution other than $(x, y, z)=(2,2,2)$ in positive integers. Most of the known results of this conjecture were proved under the assumption that 4 exactly divides $b$. The main results of this paper include the case where 8 divides $b$. One of our results treats the case where $a$ has no prime factor congruent to 1 modulo 4, which can be regarded as a relevant analogue of results due to Deng and Cohen concerning the prime factors of $b$. Furthermore, we examine parities of the three variables $x, y, z$, and give new triples $a, b, c$ such that the conjecture holds for the case where $b$ is divisible by 8 . In particular, to prove our results, we shall show an important result which asserts that if $x, y, z$ are all even, then $x / 2, y / 2, z / 2$ are all odd. Our methods are based on elementary congruence and several strong results on generalized Fermat equations given by Darmon and Merel.
\end{abstract}

2000 Mathematics subject classification: primary 11D61; secondary 11D41.

Keywords and phrases: exponential Diophantine equations, Pythagorean triples, generalized Fermat equations.

\section{Introduction}

Let $\mathbb{Z}, \mathbb{N}$ be the sets of integers and positive integers, respectively. Let $a, b, c$ be relatively prime positive integers such that $a^{2}+b^{2}=c^{2}$ with $b$ even. Then the triple $(a, b, c)$ is called a primitive Pythagorean triple. It is well known that there exist integers $m, n$ such that

$$
a=m^{2}-n^{2}, \quad b=2 m n, \quad c=m^{2}+n^{2},
$$

where $m>n>0, \operatorname{gcd}(m, n)=1, m \not \equiv n(\bmod 2)$. Clearly, the equation

$$
a^{x}+b^{y}=c^{z}, \quad x, y, z \in \mathbb{N}
$$

has the solution $(x, y, z)=(2,2,2)$.

Whether there are other solutions has been investigated by a number of authors. Sierpiński [19] showed there are no other solutions when $(a, b, c)=(3,4,5)$.

(C) 2009 Australian Mathematical Publishing Association Inc. 0004-9727/2009 \$16.00 
Jeśmanowicz [9] further showed there are no others when $(a, b, c)$ is $(5,12,13)$, $(7,24,25),(9,40,41)$ or $(11,60,61)$. He conjectured that the above equation has no solution other than $(x, y, z)=(2,2,2)$ :

Conjecture 1.1. Let $m, n$ be integers such that $m>n>0, \operatorname{gcd}(m, n)=1, m \neq \equiv$ $n(\bmod 2)$. Then the equation

$$
\left(m^{2}-n^{2}\right)^{x}+(2 m n)^{y}=\left(m^{2}+n^{2}\right)^{z}, \quad x, y, z \in \mathbb{N}
$$

has no solution other than $(x, y, z)=(2,2,2)$.

A number of other special cases of Conjecture 1.1 have since been settled. Lu [17] proved it when $n=1$. In 1965, Dem'janenko [5] extended earlier results in several papers $[10,11,18]$ by proving the conjecture to be true whenever $m-n=1$. In general, this problem has not yet been solved (see also [12, 13, 20-23]).

The first difficulty is to show that parities of $x, y, z$ are all even. For this, the simplest conditions are given by [1,7]. They assume the existence of certain prime divisors of $a$ or $b$, and, by using elementary congruences and the quadratic reciprocity law, they show that $x, y, z$ are all (or partially) even. In particular, if $x, y, z$ are all even and $2 \| m n$, then it is easily seen that $y / 2=1$, and the conclusion holds (see [8]). For example, the case $m \equiv 1(\bmod 8), n \equiv 6(\bmod 8)$ implies $(x, y, z)=(2,2,2)$. Further, some relations in $x, y, z$ are known $[6,14]$ (and see Lemma 3.1 below).

The second difficulty is to show that if we assume that $x, y, z$ are all even (so we put $x=2 X, y=2 Y, z=2 Z)$ and 4 divides $m n$, then $(X, Y, Z)=(1,1,1)$. In this case, it is important to determine the parities of $X, Y, Z$ (see Proposition 2.3 below). But even though one assumes it, it is very difficult oneself to obtain the conclusion. Most of the known results of Conjecture 1.1 concern the case where 2 exactly divides $m n$. In fact, in such a case, we can deduce much information by only elementary congruence, in particular, on determination of $y$. But, for the case where $m n$ is divisible by 4 , few results are known about the conjecture. This difficulty also appears in solving other exponential Diophantine equations $a^{x}+b^{y}=c^{z}$. In fact, most results for these concern the case where 2 exactly divides $a$ or $b$; see, for example, [1, 2, 15, 16] and [24]. Concerning this point, the following propositions have (essentially) been proved by Deng and Cohen [7, Theorems 1 and 2].

PROPOSITION 1.2. Suppose that $m$ is even with no prime factor congruent to 1 modulo 4. If $(x, y, z)$ is a solution of (1.1) and $y, z$ are even, then $(x, y, z)=(2,2,2)$.

Proposition 1.3. Suppose that $m$ is even, $n$ has no prime factor congruent to 1 modulo 4 , and $25 n>2 m$. If $(x, y, z)$ is a solution of (1.1) and $y, z$ are even, then $(x, y, z)=(2,2,2)$.

We now state our first main result.

THEOREM 1.4. Suppose that $m^{2}-n^{2}$ has no prime factor congruent to 1 modulo 4 . If $m-n$ has a prime factor congruent to 3 modulo 8 and $m \neq \equiv 1(\bmod 4)$, then Conjecture 1.1 holds. 
The assumptions in the complete version of Propositions 1.2 and 1.3 are primarily on the prime factors of $b=2 m n$. On the other hand, our assumptions in Theorem 1.4 are primarily on the prime factors of $a=m^{2}-n^{2}$, so this can be regarded as a relevant analogue of the results of Deng and Cohen. Note that the results of Deng and Cohen include the case where 4 divides $m n$, and our result also includes this case (see Example 2.12).

In Section 3, we shall examine parities of the three variables $x, y, z$ (see Lemma 3.1). As a consequence, we obtain our second main result:

THEOREM 1.5. If $m \equiv 4(\bmod 8)$ and $n \equiv 7(\bmod 16)$, or $m \equiv 7(\bmod 16)$ and $n \equiv$ $4(\bmod 8)$, then Conjecture 1.1 holds.

By Theorem 1.5, we can easily obtain pairs $m, n$ such that Conjecture 1.1 holds for the case where $m n$ is divisible by 4 .

To prove our theorems, we shall use elementary congruence and several strong results on generalized Fermat equations (see Darmon and Merel [4]). In particular, by using such strong results, we shall prove an important result which asserts that if $(x, y, z)$ is a solution of (1.1) and $x, y, z$ are all even, then $x / 2, y / 2, z / 2$ are all odd (see Proposition 2.3). This has in fact been derived under various assumptions in earlier papers, but we prove it without any of these assumptions.

In what follows, $\left(\frac{*}{*}\right)$ denotes the Jacobi symbol.

\section{Proof of Theorem 1.4}

We shall begin with the following lemmas, which are necessary to show that $x, y$ and $z$ are even.

LEMMA 2.1. Let $(x, y, z)$ be a solution of (1.1). If $m$ is even or $m$ has a divisor congruent to 3 modulo 4 , then $x$ is even.

PROOF. If $m$ is even, as is well known, by considering (1.1) mod 4, we have $2 \mid x$. If $m$ has a divisor congruent to 3 modulo 4 , say $d$, then by considering (1.1) $\bmod d$, we see that $(-1 / d)^{x}=1$. This implies that $x$ is even.

LEMMA 2.2. Let $(x, y, z)$ be a solution of (1.1). If $m+n$ has a divisor congruent to 3 modulo 8 , then $z$ is even. If $m+n$ has a divisor congruent to 7 modulo 8 , then $y$ is even. If $m-n$ has a divisor congruent to 3 or 5 modulo 8 , then $y \equiv z(\bmod 2)$.

PROOF. Let $d$ be a divisor of $m+n$. Then, from (1.1),

$$
\left(\frac{-2}{d}\right)^{y}=\left(\frac{2}{d}\right)^{z} \text {. }
$$

By the quadratic reciprocity law, we see that if $d \equiv 3(\bmod 8)$, then $(2.1)$ implies that $z$ is even, and if $d \equiv 7(\bmod 8)$, then (2.1) implies that $y$ is even.

If $d$ is a divisor of $m-n$ such that $d \equiv 3(\bmod 8)$ or $d \equiv 5(\bmod 8)$, by considering $(1.1) \bmod d$, we have $(2 / d)^{y}=(2 / d)^{z}$. This implies that $y \equiv$ $z(\bmod 2)$. 
Next, we prove an important result, derived in earlier papers under various assumptions. We prove it without any assumptions.

PROPOSITION 2.3. Let $(x, y, z)$ be a solution of (1.1). If $x, y$ and $z$ are all even, then $x / 2, y / 2$ and $z / 2$ are all odd.

Proof. First, we quote the following lemmas given by Cao and Dong [3], which play important roles in our proof. They have shown them by using the strong results from elliptic curve theory due to Darmon and Merel [4].

Lemma 2.4 [2, Lemma 9]. Suppose that $N \in \mathbb{N}$ with $N>1$. Then the equation

$$
A^{2 N}+B^{2}=C^{4}, \quad A, B, C \in \mathbb{Z}, \quad \operatorname{gcd}(A, B)=1,2 \mid A
$$

has no solution with $A B \neq 0$.

Lemma 2.5 [2, Lemma 10]. Suppose that $N \in \mathbb{N}$ with $N>1$. Then the equation

$$
A^{2 N}+B^{4}=C^{2}, \quad A, B, C \in \mathbb{Z}, \quad \operatorname{gcd}(A, B)=1
$$

has no solution with $A B \neq 0$.

By our assumption, we can put $x=2 X, y=2 Y, z=2 Z$ with $X, Y, Z \geq 1$. Since $\left\{\left(m^{2}-n^{2}\right)^{X},(2 m n)^{Y},\left(m^{2}+n^{2}\right)^{Z}\right\}$ is a primitive Pythagorean triple, we obtain

$$
\begin{aligned}
\left(m^{2}-n^{2}\right)^{X} & =s^{2}-t^{2}, \\
(2 m n)^{Y} & =2 s t, \\
\left(m^{2}+n^{2}\right)^{Z} & =s^{2}+t^{2},
\end{aligned}
$$

where $s>t>0, \operatorname{gcd}(s, t)=1, s \not \equiv t(\bmod 2)$. Since $s+t, s-t$ are relatively prime, by (2.2),

$$
s+t=u^{X}, \quad s-t=v^{X},
$$

where $u>v>0, \operatorname{gcd}(u, v)=1, u v=m^{2}-n^{2}$. Note that $u, v$ are odd since $m^{2}-n^{2}$ is odd.

Let $2^{\alpha} \| m n$ with $\alpha \geqslant 1$. Then $2^{(\alpha+1) Y} \|(2 m n)^{Y}$, that is,

$$
2^{(\alpha+1) Y-1} \| s t
$$

by (2.3).

We need the next elementary lemma.

LEMMA 2.6. $Z<2 X$ and $Z<2 Y$.

Proof. Since $s^{2}+t^{2}<\left(s^{2}-t^{2}\right)^{2}$, we know from (2.2) and (2.4) that $\left(m^{2}+n^{2}\right)^{Z}<$ $\left(m^{2}-n^{2}\right)^{2 X}$. This implies that $Z<2 X$. For the second inequality, consider (2.3), (2.4) and the inequality $s^{2}+t^{2}<(2 s t)^{2}$. 
If $X$ (or $Y$ ) is even, then, from Lemma 2.5, we must have $Y=1(X=1)$. By Lemma 2.6, this gives $Z=1$, and so $X=Y=1$ by (1.1). This is a contradiction. Hence, $X$ and $Y$ are odd. Similarly, we can show that $Z$ is odd by Lemmas 2.4 and 2.6. This completes the proof of Proposition 2.3.

REMARK 2.7. If we only treat the case $2 \alpha \neq \beta+1$ (see Section 3 for the definition of $\alpha$ and $\beta$ ), then we can show that $X, Z$ are odd without using Lemmas 2.4 and 2.5. In fact, we can prove this fact by comparing two exponents $\alpha, \beta$ as we will observe in the proof of Lemma 3.1 (also see the proof of Lemma 3.3).

Proof of TheOrem 1.4 We now invoke the assumptions of Theorem 1.4. Let $(x, y, z)$ be a solution of (1.1). We prepare several lemmas as follows.

LEMMA 2.8. $x, y$ and $z$ are all even.

PROOF. Since now $m \neq \equiv 1(\bmod 4)$, we know that $m$ is even or $m \equiv 3(\bmod 4)$. Thus, by Lemma 2.1 , we have $2 \mid x$.

Since $m+n \geq 3$ is odd, there exists an odd prime $p$ dividing $m+n$. By our assumptions, we know that $p \equiv 3(\bmod 8)$ or $p \equiv 7(\bmod 8)$, so by Lemma 2.2 , it is easily seen that $y$ and $z$ are even.

By Lemma 2.8, we may use the notation in the proof of Proposition 2.3. We prove the following lemmas.

LEMMA 2.9. $m \equiv s(\bmod 2)$ and $n \equiv t(\bmod 2)$.

Proof. By Proposition 2.3, we know that $X$ and $Z$ are odd. Then, from (2.2) and (2.4), we have $2 m^{2} \equiv 2 s^{2}(\bmod 4)$ since $m^{2}+n^{2}$ and $m^{2}-n^{2}$ are odd. This means $m \equiv s(\bmod 2)$. Clearly, $m \equiv s(\bmod 2)$ gives $n \equiv t(\bmod 2)$.

LEMMA 2.10. $\operatorname{gcd}(m-n, s+t)=1$ and $\operatorname{gcd}(m+n, s-t)=1$.

PROOF. Suppose that $\operatorname{gcd}(m-n, s+t)>1$. Then there exists a prime $p$ dividing both $m-n$ and $s+t$. Note that $p \neq 2$ and $p \equiv 3(\bmod 4)$ by our assumption. From (2.3), we have $(-1 / p)=1$ since $Y$ is odd by Proposition 2.3. However, this contradicts $p \equiv 3(\bmod 4)$. Therefore, $\operatorname{gcd}(m-n, s+t)=1$.

Similarly, we can show $\operatorname{gcd}(m+n, s-t)=1$.

It is easily seen that $u=m+n, v=m-n$ by (2.2), (2.5) and Lemma 2.10.

LEMMA 2.11. $2^{\alpha} \| s t$.

PROOF. Note that one of $m, n$ is even, the other odd. Assume that $m$ is even. Then $s$ is even and $n, t$ are odd by Lemma 2.9. From (2.5) and $2 \nmid X$, we have

$$
s=m\left(u^{X-1}-u^{X-2} v+\cdots-u v^{X-2}+v^{X-1}\right) .
$$

Since $u, v$ and $X$ are odd, $u^{X-1}-u^{X-2} v+\cdots-u v^{X-2}+v^{X-1}$ is odd. It follows from $2^{\alpha} \| m$ and (2.7) that $2^{\alpha} \| s$, and so $2^{\alpha} \| s t$ since $t$ is odd.

For the case where $n$ is even, we reach the same conclusion by a similar process. 
By (2.6) and Lemma 2.11, we have $Y=1$. Hence, Lemma 2.6 gives $Z=1$, so $X=1$. This completes the proof of Theorem 1.4.

EXAMPLE 2.12. We give the infinitely many examples of Theorem 1.4. Let $p$ be a prime such that $p>3, p \equiv 3(\bmod 8)$ and let $e, d$ be odd positive integers satisfying $p^{e}-3^{d}>2$. Then, we solve the system $m+n=p^{e}, m-n=3^{d}$, and $m=\left(p^{e}+3^{d}\right) / 2, n=\left(p^{e}-3^{d}\right) / 2$. It is easily seen that these $m, n$ satisfy the conditions of Theorem 1.4. Note that all our examples are not included in the earlier results (see Section 1). For instance, let $(p, e, d)=(11,1,1),(19,1,1)$. Then we know that $33^{x}+56^{y}=65^{z}$ and $57^{x}+176^{y}=185^{z}$ both have a unique solution $(x, y, z)=(2,2,2)$.

\section{Proof of Theorem 1.5}

In this section, we shall first examine parities of the three variables $x, y, z$. As a consequence, we have pairs $m, n$ such that Conjecture 1.1 holds for the case where $m n$ is divisible by 4 . For this purpose, we need to prepare some notation.

Note that we may assume that $n>1$ by [17]. We shall define positive integers $\alpha, \beta$ and odd positive integers $i, j$ as follows. When $m$ is even, we let

$$
m=2^{\alpha} i, \quad n=2^{\beta} j \pm 1, \quad \beta \geq 2
$$

and when $n$ is even, we let

$$
n=2^{\alpha} i, \quad m=2^{\beta} j \pm 1, \quad \beta \geq 2 .
$$

Note that if $\alpha=1$, then $2 \alpha \neq \beta+1$ since $\beta \geq 2$.

This following lemma is necessary to prove Proposition 3.2 and Theorem 1.5. Its proof includes generalizations of [21, Proposition 3] and [23, Proposition 3(2)].

LEMMA 3.1. Let $(x, y, z)$ be a solution of (1.1). If $2 \alpha \neq \beta+1$ and $y>1$, then $x \equiv z(\bmod 2)$. If $2 \alpha=\beta+1$, then $y>1$ and $x$ or $z$ is even.

PROOF. The proof is elementary. We may assume that $n>1$ by [17], and also assume that $\alpha>1$. In fact, when $\alpha=1$, by considering (1.1) $\bmod 8$, we have $( \pm 5)^{x}+4^{y} \equiv 5^{z}(\bmod 8)$. It follows that $( \pm 5)^{x} \equiv 5^{z}(\bmod 8)$ if $y>1$. This implies $x \equiv z(\bmod 2)$ if $y>1$.

First, we consider the case where $m$ is even. Since $m^{2}=2^{2 \alpha} i^{2}$ and $n^{2}=2^{2 \beta} j^{2} \pm$ $2^{\beta+1} j+1$, then

$$
\begin{aligned}
m^{2}-n^{2} & =2^{2 \alpha} i^{2}-\left(2^{2 \beta} j^{2} \pm 2^{\beta+1} j+1\right), \\
2 m n & =2^{\alpha+1} k, \quad k \text { odd }, \\
m^{2}+n^{2} & =2^{2 \alpha} i^{2}+\left(2^{2 \beta} j^{2} \pm 2^{\beta+1} j+1\right) .
\end{aligned}
$$


Case 1. $\alpha \geq \beta+1$. By considering (1.1) $\bmod 2^{\beta+2}$, we have

$$
\left(\mp 2^{\beta+1}-1\right)^{x} \equiv\left( \pm 2^{\beta+1}+1\right)^{z}\left(\bmod 2^{\beta+2}\right),
$$

and so

$$
\mp(-1)^{x-1} 2^{\beta+1} x \equiv \pm 2^{\beta+1} z\left(\bmod 2^{\beta+2}\right) .
$$

This implies that $x \equiv z(\bmod 2)$.

Case 2. $\beta+1>\alpha>1$.

Case 2.1. $\beta+1>2 \alpha$. By considering (1.1) $\bmod 2^{\beta+1}$, we have

$$
\left(2^{2 \alpha} i^{2}-1\right)^{x}+\left(2^{\alpha+1} k\right)^{y} \equiv\left(2^{2 \alpha} i^{2}+1\right)^{z}\left(\bmod 2^{\beta+1}\right),
$$

and so

$$
(-1)^{x-1} 2^{2 \alpha} x+\left(2^{\alpha+1} k\right)^{y} \equiv 2^{2 \alpha} z\left(\bmod 2^{2 \alpha+1}\right) .
$$

From this, we see that $y>1$, and so $x \equiv z(\bmod 2)$.

Case 2.2. $2 \alpha-1 \geq \beta+1>\alpha$. By considering (1.1) $\bmod 2^{\beta+2}$, we have

$$
\left(\mp 2^{\beta+1}-1\right)^{x}+\left(2^{\alpha+1} k\right)^{y} \equiv\left( \pm 2^{\beta+1}+1\right)^{z}\left(\bmod 2^{\beta+2}\right),
$$

and so

$$
\mp(-1)^{x-1} 2^{\beta+1} x+\left(2^{\alpha+1} k\right)^{y} \equiv \pm 2^{\beta+1} z\left(\bmod 2^{\beta+2}\right) .
$$

Thus, if $y=1$, then $\alpha=\beta$, so

$$
\mp(-1)^{x-1} x+k^{\prime} \equiv \pm z(\bmod 2), \quad k^{\prime} \text { odd. }
$$

This gives $x \not \equiv z(\bmod 2)$. If $y>1$, then $x \equiv z(\bmod 2)$ since $2 \alpha+2 \geq \beta+2$.

Case 2.3. $2 \alpha=\beta+1$. Note that $4 \alpha-2=2 \beta$. By considering (1.1) $\bmod 2^{4 \alpha-2}$, we have

$$
\left(\left(i^{2} \mp j\right) 2^{2 \alpha}-1\right)^{x}+\left(2^{\alpha+1} k\right)^{y} \equiv\left(\left(i^{2} \pm j\right) 2^{2 \alpha}+1\right)^{z}\left(\bmod 2^{4 \alpha-2}\right)
$$

and so

$$
(-1)^{x-1}\left(i^{2} \mp j\right) 2^{2 \alpha} x+\left(2^{\alpha+1} k\right)^{y} \equiv\left(i^{2} \pm j\right) 2^{2 \alpha} z\left(\bmod 2^{4 \alpha-2}\right) .
$$

It is clear from this congruence that $y>1$, so

$$
(-1)^{x-1}\left(i^{2} \mp j\right) x+2^{\alpha(y-2)+y} k^{\prime} \equiv\left(i^{2} \pm j\right) z\left(\bmod 2^{2 \alpha-2}\right), \quad k^{\prime} \text { odd } .
$$

This implies that $x$ or $z$ is even since $\alpha>1, y \geq 2$ and $i^{2}+j \equiv 2(\bmod 4)$ or $i^{2}-j \equiv$ $2(\bmod 4)$.

For the case where $n$ is even, we reach the same conclusion by a similar process. 
By this lemma for the case $2 \alpha \neq \beta+1$, we have the following result which can be regarded as another analogue of the results of Deng and Cohen.

Proposition 3.2. Assume that $m^{2}-n^{2}$ has no prime factor congruent to 1 modulo 4. If $2 \alpha \neq \beta+1, m-n$ has a prime factor congruent to 3 modulo 8 , then Conjecture 1.1 holds.

Proof. Let $(x, y, z)$ be a solution of (1.1). By the proof of Theorem 1.4, we know that $y, z$ are even. Thus, it suffices to show that $x$ is even. Since now $2 \alpha \neq \beta+1$ and $y>1$, by Lemma 3.1 , then $x \equiv z(\bmod 2)$. This implies $2 \mid x$.

For the case where $2 \alpha=\beta+1$, we obtain the following lemma, which does not need assumptions on prime factors of $m^{2}-n^{2}$ or $2 m n$.

LEMMA 3.3. Assume that $2 \alpha=\beta+1$. If $(x, y, z)$ is a solution of (1.1), $y$ is even and $x \equiv z(\bmod 2)$, then $(x, y, z)=(2,2,2)$.

PROOF. The proof is similar to the case $2 \alpha=\beta+1$ in Lemma 3.1. By Lemma 3.1, we know that $x, y$ and $z$ are all even. Thus, we may use the notation in Section 2 . First, we consider the case where $m$ is even. We know that $s$ is even and $t$ is odd by Lemma 2.9. Note that $\alpha>1$ and

$$
2^{2(\alpha+1) Y-1} \| 2 s^{2}
$$

by (2.6). From (2.2) and (2.4),

$$
\left(2^{2 \alpha} i^{2}-\left(2^{4 \alpha-2} j^{2} \pm 2^{2 \alpha} j+1\right)\right)^{X}+\left(2^{2 \alpha} i^{2}+\left(2^{4 \alpha-2} j^{2} \pm 2^{2 \alpha} j+1\right)\right)^{Z}=2 s^{2} .
$$

Then, by considering (3.1) $\bmod 2^{4 \alpha-2}$, we have

$$
\left(\left(i^{2} \mp j\right) 2^{2 \alpha}-1\right)^{X}+\left(\left(i^{2} \pm j\right) 2^{2 \alpha}+1\right)^{Z} \equiv 2^{2(\alpha+1) Y-1} s^{\prime}\left(\bmod 2^{4 \alpha-2}\right), \quad s^{\prime} \text { odd },
$$

and so

$$
(-1)^{X-1}\left(i^{2} \mp j\right) 2^{2 \alpha} X+\left(i^{2} \pm j\right) 2^{2 \alpha} Z \equiv 2^{2(\alpha+1) Y-1} s^{\prime}\left(\bmod 2^{4 \alpha-2}\right) .
$$

Thus,

$$
(-1)^{X-1}\left(i^{2} \mp j\right) X+\left(i^{2} \pm j\right) Z \equiv 2^{2 \alpha(Y-1)+(2 Y-1)} s^{\prime}\left(\bmod 2^{2 \alpha-2}\right) .
$$

Now suppose that $Y>1$. Then

$$
(-1)^{X-1}\left(i^{2} \mp j\right) X+\left(i^{2} \pm j\right) Z \equiv 0\left(\bmod 2^{2 \alpha-2}\right) .
$$

This implies that $X$ or $Z$ is even since $\alpha>1$ and $i^{2}+j \equiv 2(\bmod 4)$ or $i^{2}-j \equiv$ $2(\bmod 4)$. But this contradicts Proposition 2.3. Thus, $Y=1$, and so $X=Z=1$ by Lemma 2.6.

For the case where $n$ is even, we reach the same conclusion by a similar process.

Using this lemma, we shall prove Theorem 1.5. 
Proof of Theorem 1.5. Assume the cases $m \equiv 4(\bmod 8)$ and $n \equiv 7(\bmod 16)$, or $m \equiv 7(\bmod 16)$ and $n \equiv 4(\bmod 8)$. Note that $\alpha=2, \beta=3$. In particular, $2 \alpha=\beta+1$. For both cases, we know $m \neq \equiv 1(\bmod 4)$. Thus, by Lemma 2.1 , we see that $x$ is even. For the case $m \equiv 4(\bmod 8)$ and $n \equiv 7(\bmod 16)$, we know that $m+n \equiv 3(\bmod 8)$ and $m-n \equiv 5(\bmod 8)$. Hence, by Lemma 2.2, we see that $y$ and $z$ are even. For the case $m \equiv 7(\bmod 16)$ and $n \equiv 4(\bmod 8)$, it is similarly seen that $y$ and $z$ are even. Therefore, $x, y$ and $z$ are all even. Thus, by Lemma 3.3, $(x, y, z)=(2,2,2)$.

From Theorem 1.5, we can easily obtain infinitely many examples which are not included in earlier results (see Section 1). For instance, let $(m, n)=(7,4),(23,12)$. Then we know that $33^{x}+56^{y}=65^{z}$ and $85^{x}+552^{y}=673^{z}$ each have a unique solution $(x, y, z)=(2,2,2)$.

\section{Acknowledgements}

The author is grateful to Professors Hirofumi Tsumura and Isao Wakabayashi for their valuable comments and suggestions, and also to Dr Yohei Tachiya for his important comment on Example 2.12. In addition, the author wishes to thank the referee for valuable suggestions and constructive remarks which improved the paper.

\section{References}

[1] Z. F. Cao, 'A note on the Diophantine equation $a^{x}+b^{y}=c^{z}$, Acta Arith. 91 (1999), 85-93.

[2] Z. F. Cao and X. L. Dong, 'On the Terai-Jeśmanowicz conjecture', Publ. Math. Debrecen 61 (2002), 253-265.

[3] — 'An application of a lower bound for linear forms in two logarithms to the TeraiJeśmanowicz conjecture', Acta Arith. 110 (2003), 153-164.

[4] H. Darmon and L. Merel, 'Winding quotients and some variants of Fermat's last theorem', J. Reine. Angew. Math. 490 (1997), 81-100.

[5] V. A. Dem'janenko, 'On Jeśmanowicz' problem for Pythagorean numbers', Izv. Vyš̌. Učebn. Zaved. Matematika 48 (1965), 52-56 (in Russian).

[6] M.-J. Deng and G. L. Cohen, 'On the conjecture of Jeśmanowicz concerning Pythagorean triples', Bull. Aust. Math. Soc. 57 (1998), 515-524.

[7] - 'A note on a conjecture of Jeśmanowicz', Colloq. Math. 86 (2000), 25-30.

[8] Y.-D. Guo and M.-H. Le, 'A note on Jeśmanowicz conjecture concerning Pythagorean numbers', Comment. Math. Univ. St. Pauli 44 (1995), 225-228.

[9] L. Jeśmanowicz, 'Several remarks on Pythagorean numbers', Wiadom. Mat. 1 (1955/1956), 196-202 (in Polish).

[10] C. Ko, 'On Pythagorean numbers', Sichuan Daxue Xuebao 1 (1958), 73-80 (in Chinese).

[11] ' 'On Jeśmanowicz conjecture', Sichuan Daxue Xuebao 2 (1958), 31-40 (in Chinese).

[12] M. H. Le, 'A note on Jeśmanowicz conjecture', Colloq. Math. 69 (1995), 47-51.

[13] ' 'On Jeśmanowicz conjecture concerning Pythagorean numbers', Proc. Japan Acad. Ser. A Math. Sci. 72 (1996), 97-98.

[14] - 'A note on Jeśmanowicz' conjecture concerning Pythagorean triples', Bull. Aust. Math. Soc. 59 (1999), 477-480.

[15] 'A conjecture concerning the exponential diophantine equation $a^{x}+b^{y}=c^{z}$, Acta Arith. 106 (2003), 345-353. 
[16] - 'A conjecture concerning the pure exponential equation $a^{x}+b^{y}=c^{z}$, Acta Math. Sinica, English Series 21 (2004), 943-948.

[17] W. T. Lu, 'On the Pythagorean numbers $4 n^{2}-1,4 n$ and $4 n^{2}+1$ ', Acta Sci. Natur. Univ. Szechuan 2 (1959), 39-42 (in Chinese).

[18] V. D. Podsypanin, 'On a property of Pythagorean numbers', Izv. Vyssh. Uchebn. Zaved. Mat. 4 (1962), 130-133 (in Russian).

[19] W. Sierpiński, 'On the equation $3^{x}+4^{y}=5^{z}$, Wiadom. Mat. 1 (1955/1956), 194-195 (in Polish).

[20] K. Takakuwa, 'A remark on Jeśmanowicz conjecture', Proc. Japan Acad. Ser. A Math. Sci. 72 (1996), 109-110.

[21] K. Takakuwa and Y. Asaeda, 'On a conjecture on Pythagorean numbers', Proc. Japan Acad. Ser. A Math. Sci. 69 (1993), 252-255.

[22] - 'On a conjecture on Pythagorean numbers II', Proc. Japan Acad. Ser. A Math. Sci. 69 (1993), 287-290.

[23] 'On a conjecture on Pythagorean numbers III', Proc. Japan Acad. Ser. A Math. Sci. 69 (1993), 345-349.

[24] N. Terai, 'Applications of a lower bound for linear forms in two logarithms to exponential Diophantine equations', Acta Arith. 86 (1999), 17-35.

TAKAFUMI MIYAZAKI, Department of Mathematics and Information Sciences, Tokyo Metropolitan University, 1-1, Minami-Ohsawa, Hachioji, Tokyo 192-0397, Japan

e-mail: miyazaki-takafumi@ed.tmu.ac.jp 